Meta

Journal des tradlucteurs

Translators' Journal

\title{
Initiation à la traduction par la révision
}

\section{Bernard Joyal}

Volume 14, numéro 2, juin 1969

URI : https://id.erudit.org/iderudit/003272ar

DOI : https://doi.org/10.7202/003272ar

Aller au sommaire du numéro

Éditeur(s)

Les Presses de l'Université de Montréal

ISSN

0026-0452 (imprimé)

1492-1421 (numérique)

Découvrir la revue

Citer cet article

Joyal, B. (1969). Initiation à la traduction par la révision. Meta, 14(2), 98-100. https://doi.org/10.7202/003272ar d'utilisation que vous pouvez consulter en ligne.

https://apropos.erudit.org/fr/usagers/politique-dutilisation/ 


\section{initiation à la traduction par la révision}

On entend parfois des gens demander quel est le meilleur moyen de devenir traducteur. La réponse peut varier d'un individu à l'autre, car traduire est forcément une occupation empreinte de subjectivité. Néanmoins, quels que soient ses antécédents, ses aptitudes ou ses goûts personnels, celui qui aspire à devenir traducteur peut suivre des cours du soir ou fréquenter une université qui offre des cours réguliers d'une durée de deux ou trois ans. Une autre avenue ouverte aux intéressés, celle qu'ont prise la majorité de ceux qui sont aujourd'hui dans la profession, leur permet de commencer au bas de l'échelle et de se former à cette discipline en travaillant dans un service de traduction public ou privé.

Qu'il soit ou non détenteur d'un diplôme de traduction, le novice n'a qu'un seul moyen d'acquérir de l'expérience dans la carrière de son choix: faire de la traduction régulièrement pendant bon nombre d'années. On l'affecte à un service où tous ses textes sont soigneusement revus par des collègues qui eux-mêmes ont travaillé longtemps avant d'accumuler le bagage linguistique et les connaissances générales nécessaires pour être réviseur.

Il est tout à fait juste de dire que le meilleur apprentissage est la pratique constante sous direction experte. Mais, faut-il nécessairement que les trois ou quatre premières années soient consacrées exclusivement à traduire, sans plus ? Tout comme l'écolier apprend à bien manier sa langue maternelle par la lecture assidue de bons auteurs, le traducteur stagiaire ne pourrait-il pas faire des progrès encore plus rapides si l'occasion lui était parfois donnée d'examiner attentivement les textes de ses collègues, surtout de ceux qui ont déjà quelques années d'expérience en traduction? Autrement dit, pourquoi la formation et le perfectionnement du traducteur ne consisteraient-ils pas en partie à faire du travail de révision?

À première vue, il peut sembler quelque peu ridicule de donner au novice le texte d'un traducteur chevronné et de lui demander d'en faire la révision. On sait déjà qu'il faut avoir passé des mois et des mois à peiner sur toutes sortes de 
traductions avant d'arriver à se perfectionner assez pour posséder la compétence qu'exigent les fonctions de réviseur. Est-il raisonnable alors de s'attendre qu'un traducteur non rompu à sa profession soit capable de faire des révisions constructives? Certes, la question n'a rien d'injustifié, mais si l'on se rappelle que le but visé serait la formation par la révision plutôt que la révision en tant que telle, l'idée mérite qu'on s'y arrête.

Le traducteur en herbe ne saurait guère oublier qu'il a sous les yeux le texte traduit par une personne qui n'en est plus à ses premières armes. Au début, il fera preuve d'une circonspection telle qu'il se limitera à lire attentivement les deux versions, s'en tenant à relever les fautes d'orthographe ou de ponctuation. Par mesure de prudence, il faudra évidemment que le texte soit vérifié par un réviseur attitré. Une fois que celui-ci aura terminé son travail et que la traduction sera considérée comme définitive, le traducteur et l'aspirant prendront quelques minutes pour discuter les corrections apportées au texte. C'est alors que le stagiaire aura le plus à gagner, car il lui sera loisible de poser des questions et de se faire expliquer le pourquoi de telle ou telle modification, de s'instruire sur les nuances et les acceptions de différents mots, d'apprendre comment procéder pour faire des recherches ou se servir des ouvrages de référence; bref, il s'initiera à la traduction.

Après quelques jours pendant lesquels il n'aura fait rien d'autre que de la traduction (mettant en pratique les quelques conseils qu'il aura pu retenir de son envolée initiale comme réviseur), on lui passera un autre texte à revoir. Même procédé: révision, puis discussion. Et le cycle recommencera.

Quelques mois seulement s'écouleront avant qu'on note des progrès sensibles dans les traductions du stagiaire. En travaillant ainsi en étroite collaboration avec d'autres plus versés que lui dans la discipline, il apprendra rapidement à déceler les difficultés, à éviter les traquenards et les grossiers contresens, à faire un emploi judicieux des nombreux dictionnaires et lexiques à sa disposition. Les quelques travaux de révision qu'on lui aura confiés auront suffi à lui démontrer que la tâche du réviseur est rarement aisée et souvent ingrate; si limitée qu'elle aura été, son expérience lui aura enseigné que le traducteur se doit de développer des dispositions constructives à l'égard du réviseur. Sa réaction devant les difficultés sera de s'appliquer à les résoudre au mieux de ses connaissances plutôt que de s'en remettre aveuglément, pour ne pas dire lâchement, au réviseur. Contrairement à l'attitude qu'affichent parfois certains traducteurs, il sera enclin à faire bénéficier le réviseur de ses recherches en notant les références sur sa copie au lieu de n'en rien faire, espérant secrètement qu'il pourra prendre en défaut le réviseur pressé par un surcroît de travail. En un mot, il s'intégrera plus facilement à l'équipe, fort de savoir qu'il se perfectionnera davantage s'il sait tirer profit de l'expérience acquise par ses collègues, qu'ils soient traducteurs ou réviseurs.

Évidemment, cette méthode de formation nécessite qu'un même texte soit revu par deux personnes, le stagiaire et un des réviseurs habituels. C'est dire que, jusqu'à un certain point, ils font double emploi. Pour cette raison, elle ne serait pas pratique ni recommandable lorsqu'il s'agit de travaux urgents qui ne peuvent souffrir aucun retard. En revanche, il y a toujours de ces textes qu'il faut traduire «le plus tôt possible " et qui ne sont pas de nature à bouleverser la tournure des 
événements, même s'ils sont rendus demain plutôt qu'aujourd'hui. Les quelques heures supplémentaires que nécessite la double révision peuvent être extrêmement profitables au stagiaire. Quant au réviseur, il ne mettra guère plus de temps à revoir le texte déjà vérifié par son jeune collègue qu'il ne l'aurait fait si ce dernier n'y avait pas mis la main. D'ailleurs, il n'est pas du tout inconcevable que la tâche du réviseur en soit rendue plus facile, particulièrement lorsque le texte comporte de longues listes de noms ou de chiffres que le stagiaire est tout aussi capable de vérifier consciencieusement; le deuxième réviseur se trouve ainsi libéré de la nécessité de s'attarder sur des détails fastidieux et il peut consacrer son temps précieux à des tâches plus productives.

Après quelques mois, le stagiaire n'est plus stagiaire, il est traducteur. Parce qu'il traduit, il est meilleur réviseur; parce qu'il révise, il est meilleur traducteur. Tout compte fait, sa formation de traducteur aura été à la fois moins superficielle et plus rapide, sa contribution à l'équipe, vraiment appréciable, son potentiel, tant comme traducteur que comme réviseur, plein de promesses.

Bien sûr, il ne saurait pour cela se targuer d'être passé maître en traduction ou en révision, mais il sera sur la bonne voie. Sa valeur n'aura plus qu'à attendre un peu le nombre des années...

BERNARD JOYAL 